\title{
What ancient scalar satellite data can tell us about the 1969 geomagnetic jerk?
}

\author{
Y. Yahiat ${ }^{1}$, M. Hamoudi ${ }^{1}$, and M. Mandea ${ }^{2}$ \\ ${ }^{1}$ University of Sciences and Technology Houari Boumediène, FSTGAT, Geophysics Department, Bab-Ezzouar, Alger \\ ${ }^{2}$ Helmholtz-Zentrum Potsdam, Deutsches GeoForschungsZentrum (GFZ), Germany
}

(Received August 8, 2008; Revised January 20, 2009; Accepted April 8, 2009; Online published August 31, 2009)

\begin{abstract}
The linearity of the secular variation of the Earth's magnetic field is irregularly broken by sudden changes in its trend, known as geomagnetic jerks. Detecting these events in the temporal variations of the magnetic field components continues to be an exciting topic, mainly recently when their investigation rely not more only on observatory data, but as well as on measurements provided by satellites. We have also been interested by these magnetic events, and one of the central aims of our work has been to determine whether the satellite scalar data, obtained in the sixties and early seventies are able to reveal the existence of such a geomagnetic jerk, previously detected around 1969, in observatory annual or monthly means. For this purpose, we have used available OGO2, OGO4 and OGO6 satellite intensity data covering the period 1965-1971. Another motivation of re-processing and analyzing these old satellite datasets has been to better estimate their role in the global core field modeling, especially in describing the secular variation. Our results indicate that these ancient magnetic satellite datasets contain valuable information to characterize the secular variation over the time-span they are available, and allow to detect the geomagnetic jerk around 1969.
\end{abstract}

Key words: Core magnetic field, secular variation, geomagnetic jerk, scalar data, POGO.

\section{Introduction}

The measured geomagnetic field is the result of internal and external sources, with respect of the Earth's surface. Indeed, the magnetic field, at an arbitrary location at the Earth's surface, is the sum of the core and lithospheric fields, as internal contributions, and of the ionospheric and magnetospheric fields, as external contributions. Moreover, to these main sources one can add the magnetic field induced in the crust and the upper mantle by geomagnetic field external variations (see for more details Mandea and Purucker, 2005). At the satellite altitude, the separation between internal and external sources is no more available, as the ionosphere contribution is partially seen by a satellite as an internal source. The core field, also known as main field, is generated in the outer fluid core, formed mainly of iron, thus very conductive. The fluid motion generates electric currents, which maintain a magnetic field. Such a phenomenon is known as a self-sustaining dynamo. The magnitude of this field, at the Earth's surface, ranges between approximately 30000 nanoTeslas (nT) in the equatorial regions (with minimum values around $20000 \mathrm{nT}$ for the South Atlantic Anomaly), and about 70000 nT in the magnetic pole areas (Mandea et al., 2007).

Even if our interest is not to characterize all magnetic sources, understanding their role and variations is crucial when analyzing core field evolution. The lithospheric field, also known as magnetic anomaly field, is caused by heterogeneity magnetization of rocks in the crust and upper

Copyright (c) The Society of Geomagnetism and Earth, Planetary and Space Sciences (SGEPSS); The Seismological Society of Japan; The Volcanological Society of Japan; The Geodetic Society of Japan; The Japanese Society for Planetary Sciences; TERRAPUB. mantle. Its magnitude varies usually from a few nT to some hundreds nT, with the strongest magnetic anomalies reaching some thousands nT (Hamoudi et al., 2007), and depends on the nature and thickness of the magnetic layers. Its contribution to the internal field is, however, significantly lower than the core field ones, and it is considered constant on historical time-scales. Ionospheric and magnetospheric fields are produced by currents circulating in the ionosphere and magnetosphere, respectively. These two regions, surrounding the neutral atmosphere and the Earth, are disturbed by solar wind, directly linked to the solar activity. The external field variations are of order of a few tens of nT during quiet periods and can reach several thousands of nT during magnetic storms (Gonzalez et al., 1994). These variations are characterized by magnetic activity indices, as $D_{\text {st }}$ (swdcwww.kugi.kyoto-u.ac.jp/) and $K_{\mathrm{p}}$ (gfzpotsdam.de/pb2/pb23/GeoMag/niemegk/kp_index/).

The temporal variation of the core field, called secular variation is estimated as the first derivative of the geomagnetic field. Once in a while, the secular variation reveals abrupt changes in its trend or jumps, called geomagnetic jerks (Courtillot et al., 1978; Mandea et al., 2000, and references therein). The origin of these jerks remains unknown although many authors attempted to identify the processes that are responsible (Le Huy et al., 2000; Bloxham et al., 2002; Olsen and Mandea, 2007), and which are linked to the core dynamics (Chambodut et al., 2007).

Studies of the temporal behavior of the magnetic field have usually been made by using the geomagnetic observatory data, and more recently satellite data, leading to a better understanding of the geomagnetic jerks. The wavelet analysis of monthly means (Alexandrescu et al., 1995, 1996) had better characterized the geomagnetic jerks, without a pri- 
ori information on their temporal and spatial distribution. Sabaka et al. (2002, 2004) have used combined observatory and satellite data in continuous field models, so-called "comprehensive models", the last available being CM4, and discuss the secular variation seen by these models. Other authors have then deepened this idea (e.g. Chambodut and Mandea, 2005) to examine the occurrence dates of geomagnetic jerks after 1960, starting date for the CM4 model. A new approach proposed by Mandea and Olsen (2006) shows that the temporal variations, at the scale of a month, derived from observatory data are similar to those derived from the CHAMP satellite data. In a second study, Olsen and Mandea (2007) have established a grid of virtual observatories, covering the whole Earth. The temporal variations of the three magnetic field components were studied for all defined locations. Two interesting conclusions have been drawn from this study: first, that the geomagnetic jerks can be detected in the satellite data and second, that these phenomena may be rather regional than global.

One main objective of our work is to study whether the satellite scalar data obtained in the mid-sixties and early seventies are able to evidence the existence of such geomagnetic jerks. Hence, we have used the available satellite data covering that period: data from the American satellites POGO (Polar Orbiting Geomagnetic Observatory), which carried magnetometers (Cain et al., 1966, 1967). These satellite missions recorded intensity data of the magnetic field from October 1965 to April 1971, only. Another goal of re-analyzing these old satellite datasets is to estimate their contribution in the global modeling of the core field, for a better description of the secular variation for this period.

After introducing the used data, we present in what follows the applied methods for studying the temporal variation of the field. For this, we explain an approach to minimize the influence of the large disparity in time and altitude of available data, when investigating the field behavior. Data from ground-based observatories and satellites have been compared for a number of locations. An indirect evaluation of the secular variation is then presented, obtained from spherical harmonics global field models. We present, for each of the selected locations, an annual-basis field variation derived from the spherical harmonics model based on the POGO data, only. Comparison of these temporal variations to the equivalent variations computed from the continuous CM4 model of Sabaka et al. (2004) is thereafter shown. A map derived for the 1965-1971 time-interval, highlighting on a global scale the variation of intensity field calculated on a regular grid is used for our concluding remarks.

\section{Data}

The present study is based on two data types: those gathered by the U.S. satellites OGO-2, OGO-4 and OGO-6 of the POGO (Polar Orbiting Geomagnetic Observatory) series, and those provided by the chosen ground geomagnetic observatories.

\subsection{Satellite data}

The American satellite POGO mission began in 1965 and included a total number of six satellites. Several studies have been conducted using these data (e.g. Kane, 1973; Kim and King, 1999). However, over six satellites, only three provided reliable magnetic data.

The satellite intensity measurements, which we are interested in, cover the time period between October 1965 and February 1971. These data are available from different satellites and for different time intervals. The OGO-2 satellite with an inclination of $87.3^{\circ}$ sampled the magnetic field from October 14, 1965 to October 02, 1967. It was followed by OGO-4 mission from July 29, 1967 to January 19, 1969, with an inclination of $86^{\circ}$. The last satellite, OGO-6, with an inclination of $82^{\circ}$, measured the field from June 6, 1969 to April 26, 1971. Unfortunately, the latter period was not fully covered and the data series available exhibit several gaps, sometimes reaching a few days, to a maximum one of some four months. These satellites sampled the intensity of the Earth's magnetic field every 0.5 seconds for OGO-2 and OGO-4, and every 0.288 seconds for OGO- 6 . The whole POGO database reaches several millions measurements.

The POGO orbits were elliptic, with altitudes ranging, for example, from $413 \mathrm{~km}$ at the perigee to $1510 \mathrm{~km}$ at the apogee in the case of OGO-2. Figure 1 shows the temporal distribution of the OGO-2 altitude data and illustrates their large altitudinal dispersion. The same remark holds true for data distributions of the other two satellites. Accordingly, in the case of OGO-4, the altitude range extends from 410 to $910 \mathrm{~km}$, while for OGO-6, from 400 to $1100 \mathrm{~km}$.

\subsection{Observatory data}

The ground dataset is based on monthly means, computed as the means of all hours of a day and all days of a month (Alexandrescu, 1998; Chulliat and Telali, 2007). In order to compare the obtained results with POGO satellite data, we have selected observatory data over the 19651971 time interval. Moreover, still for comparative purposes, but this time with the study of Mandea and Olsen (2006), we choose to examine the temporal variations of the same three observatories, i.e. Niemegk (NGK) observatory in Germany, Hermanus (HER) in South Africa, and Kakioka (KAK) in Japan. Three more observatories, located at different latitudes, are also analyzed and discussed here: Alibag (ABG) in India, Fredericksburg (FRD) in the USA, and Huancayo (HUA) in Peru.

\section{Methods and Results}

We present the two approaches used to assess the secular variation. The first one, direct, from temporal series of observatory and satellite data and the second one, indirect, through global spherical harmonics field models, based on POGO data, only.

\subsection{Direct secular variation study: temporal series from observatories and scalar satellites}

To carry out this study we have considered the approach proposed by Mandea and Olsen (2006) to build "virtual observatory" series from satellite available data over the real observatory locations. First, we have selected data in a $2^{\circ} \times 2^{\circ} \times \Delta h$ volume blocks with respect of the latitude, longitude and satellite altitude variations, centered on the six chosen observatory positions, ABG, FRD, HER, HUA, KAK and NGK. The analysis of the data distribution, as a function of altitude, shows, as expected, great discrepancy 


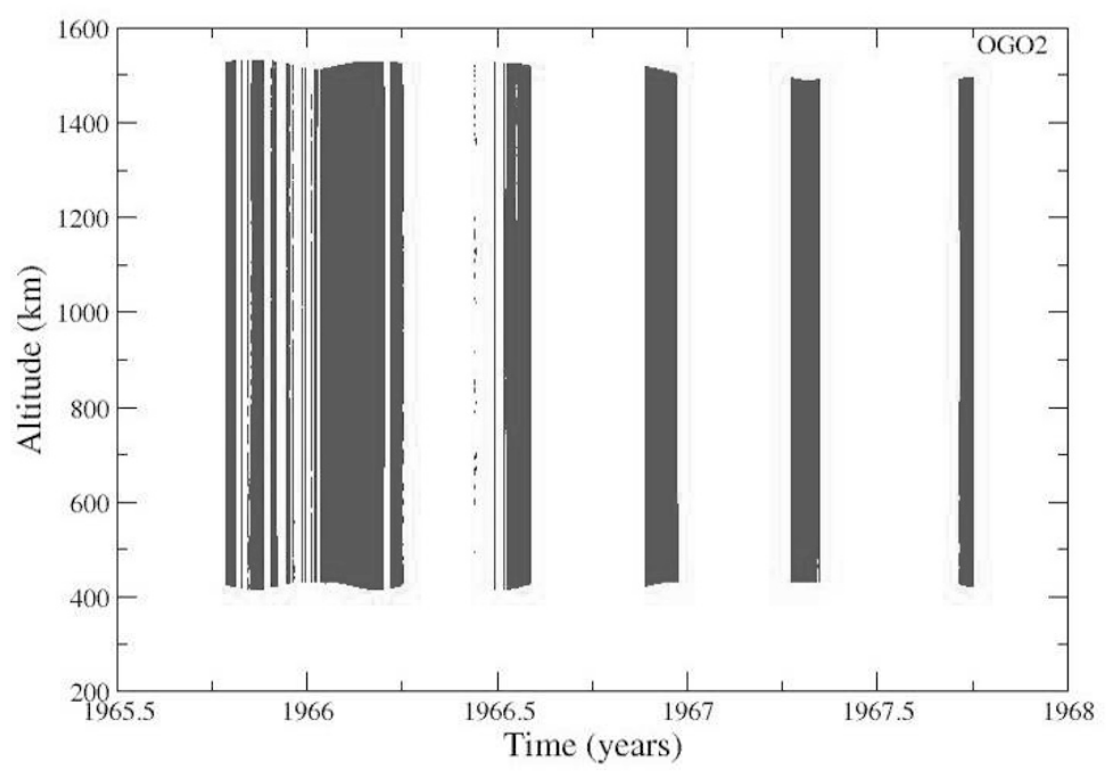

Fig. 1. Temporal distribution of the OGO-2 satellite altitude, between 1965 and 1967.

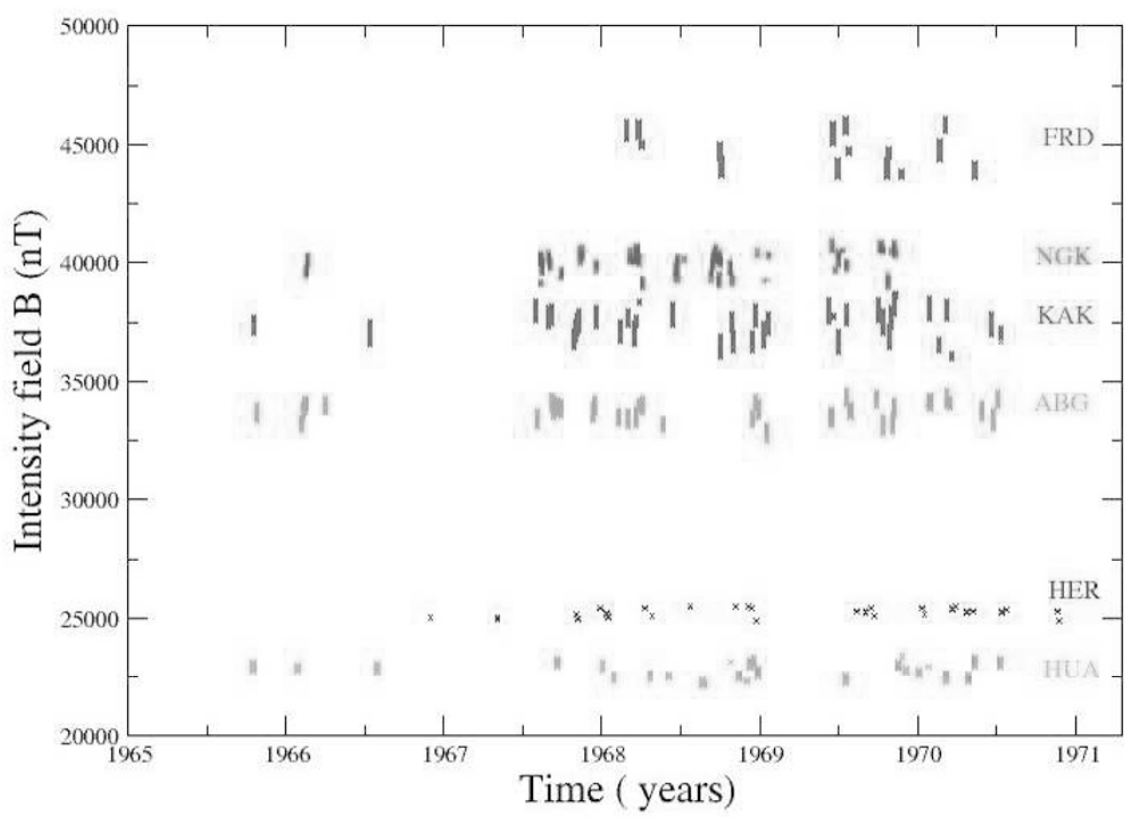

Fig. 2. Total magnetic field derived from POGO satellite data. Measurements, at the six selected observatory positions, are selected for $100 \mathrm{~km}$ altitude bandwidth.

in the magnetic field magnitude, of about $18000 \mathrm{nT}$ for the FRD position, $14000 \mathrm{nT}$ for ABG, more than $10000 \mathrm{nT}$ for KAK and NGK positions and around $8000 \mathrm{nT}$ and $6000 \mathrm{nT}$ for HUA and HER, respectively. This is not surprising when one considers the satellite elliptical trajectories that induce some $600 \mathrm{~km}$ in altitude variations.

To minimize the influence of variation of this spatial parameter in our temporal study, we have divided the three satellite datasets in several altitudinal bands of equal $\Delta h$ height. Several trials showed that a $\Delta h$ of $100 \mathrm{~km}$ is manda- tory to obtain a sufficiently dense data blocks. In addition, let us note that the comparison of data distributions for different bands shows that the lowest altitude band presents a better distribution for the six chosen observatories.

The evolution of the geomagnetic field intensity for the chosen band and for the six observatory locations is shown in Fig. 2. In addition to the data lack during long time intervals, we also observe large variation in the field $\mathbf{B}$, from some $650 \mathrm{nT}$ for the HER observatory, up to $2700 \mathrm{nT}$ for $\mathrm{KAK}$, respectively. With such variations in the field, due 


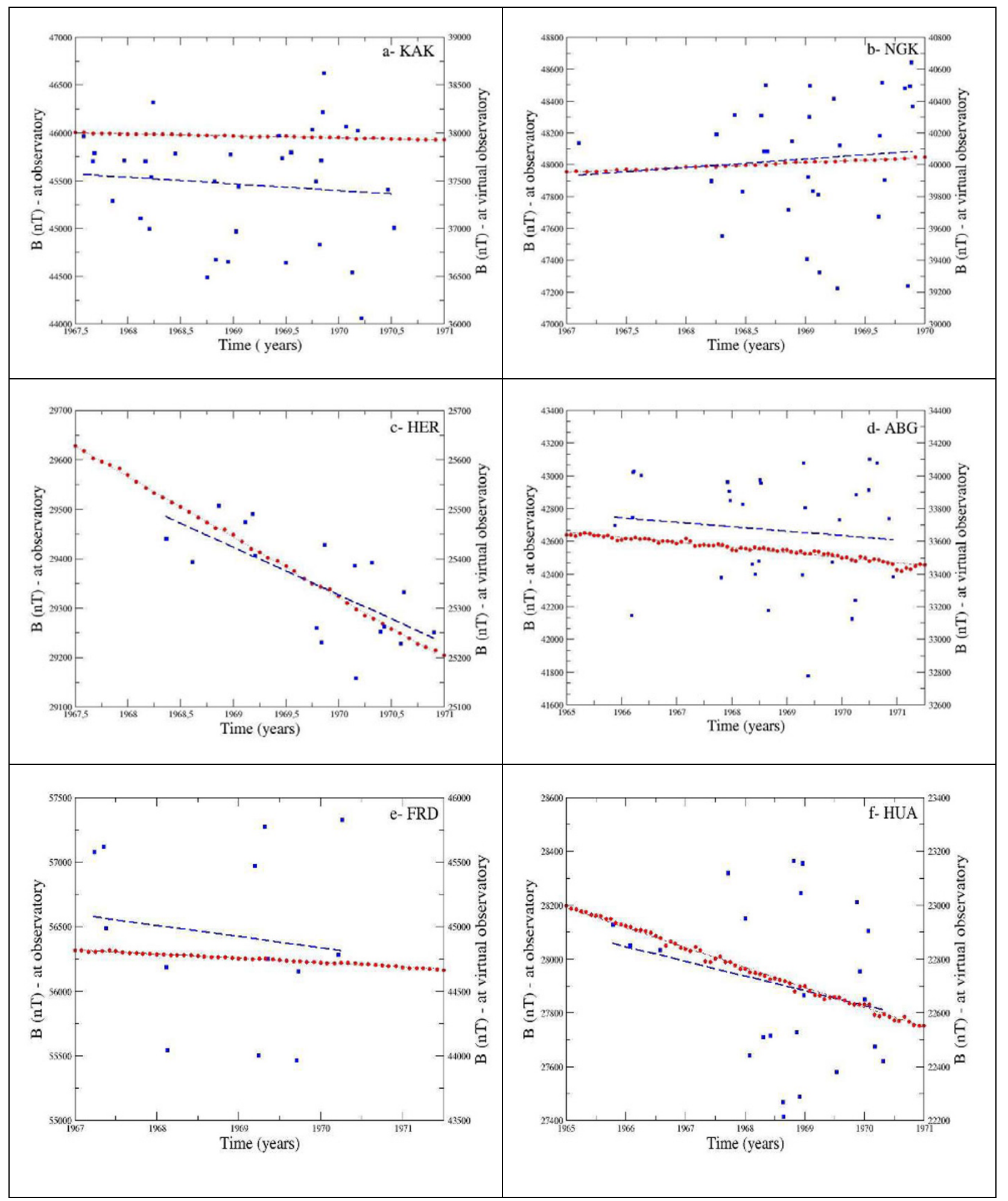

Fig. 3. Observatory monthly means (solid circles and left-side axis) and POGO data taken in an altitudinal band $\Delta h=100 \mathrm{~km}$ (solid squares and right-side axis) for the corresponding positions: (a) KAK, (b) NGK, (c) HER, (d) ABG, (e) FRD, (f) HUA. The estimated linear regressions for POGO (dashed lines) and observatory (dotted lines) data are also plotted.

to the measurement locations, a question arises about the quality of the estimated secular variation.

Considering the non-regular time sampling data, it is appropriate to average the intensity field $\mathbf{B}$ along each orbit, independently. Then, we have estimated the temporal variations of the geomagnetic field intensity, averaged per orbit, in the same band of altitude. These mean values are then compared to the monthly means of the intensity of the magnetic field from the chosen observatories. The trends of the two datasets, from the monthly means provided by the selected observatories and the POGO means for the corresponding positions, are illustrated in Figs. 3(a) to 3(f) for KAK, NGK, HER, ABG, FRD, and HUA, respectively. We can notice the same behavior of the temporal tendencies be- 
Table 1. Statistics for different POGO models.

\begin{tabular}{|c|c|c|c|c|c|}
\hline \multirow{2}{*}{ Satellite } & \multirow{2}{*}{ Iteration number } & \multirow{2}{*}{$\operatorname{RMS} \chi(\mathrm{nT})$} & \multicolumn{3}{|c|}{ Number of data points } \\
\hline & & & & & Equator \\
\hline OGO-2 (1965) & 3 & 25 & & 994042 & 1414 \\
\hline OGO-2 (1966) & 2 & 40 & & 1655227 & 1426 \\
\hline OGO-2 (1967) & 2 & 264 & & 110913 & 1384 \\
\hline OGO-4 (1967) & 2 & 27 & & 1473382 & 1384 \\
\hline OGO-4 (1968) & 2 & 30 & & 1697551 & 1390 \\
\hline OGO-4 (1969) & 2 & 29 & & 213275 & 1407 \\
\hline OGO-6 (1969) & 2 & 38 & & 66779846 & 1407 \\
\hline OGO-6 (1970) & 2 & 33 & & 4063022 & 1422 \\
\hline OGO-6 (1971) & 2 & 19 & $\left(\right.$ for $\left.K_{\mathrm{p}}=1\right)$ & 153358 & 1422 \\
\hline
\end{tabular}

tween observatory data trends and those of the POGO satellite data, for the investigated observatory data. This similarities are underlined by the estimated linear regressions, also plotted on Fig. 3. This comparable behavior for ground and virtual observatory intensity data makes it possible to consider the approach proposed by Olsen and Mandea (2007) and then to build a global grid points for which the scalar secular variation is estimated. This is done in slightly different way that in the above study, and is discussed at the end of this section.

\subsection{Indirect secular variation study: estimation from core field models}

3.2.1 Global modeling The use of global field models, continuous in time, like the so-called "Comprehensive Model" of Sabaka et al. (2004) has allowed to investigate the geomagnetic jerks with a total coverage of the Earth's surface (e.g. Chambodut and Mandea, 2005). So, we have taken into account this possibility to explore the secular variation, by computing annual core magnetic field models.

Let's briefly recall that in a region free of magnetic sources, the Earth's magnetic field is Laplacian and derives from a harmonic scalar potential $V$. Indeed, the magnetic field is the spatial gradient of the potential, the two functions being linked by: $\mathbf{B}=-\nabla V$. Since Gauss, $V$ is traditionally developed on the basis of spherical harmonics functions. Thus, if $(r, \theta, \phi)$ are the geocentric spherical coordinates of any point in the considered domain, the internal potential $V(r, \theta, \phi)$ is given by the following equation:

$$
\begin{aligned}
V(r, \theta, \phi)= & a \sum_{n=1}^{N_{\max }}\left(\frac{a}{r}\right)^{n+1} \sum_{m=0}^{n}\left\{g_{n}^{m} \cos m \phi+h_{n}^{m} \sin m \phi\right\} \\
& \cdot P_{n}^{m}(\cos \theta),
\end{aligned}
$$

where, $a$ is the radius of the reference sphere, $n$ and $m$ are the degree and the order of development, $g_{n}^{m}$ and $h_{n}^{m}$ the Gauss coefficients and $P_{n}^{m}(\cos \theta)$ Legendre associated functions semi-normalised under Schmidt (Chapman and Bartels, 1940). Let us underline that in the above expression the external contributions are not considered. Moreover, this potential expansion is not strictly valid at the satellite altitudes, where non-Laplacian fields also exist. In order to minimize these contributions a dedicated data selection is needed.

Modeling the geomagnetic field leads to mathematically determine the Gauss coefficients which describe it. Numerically, these coefficients are usually determined from measurements by inverse methods. We describe here, very briefly, the applied method, the least squares one, which aims to minimize the square of the differences between the measured value $B_{i}^{\text {mes }}$ of the field and the predicted value $B_{i}^{\text {mod }}$ localized at the same point in space, for all the observations $K$. To each measurement $i$ is assigned a weight $\omega_{i}$. Thus, solving the inverse problem is to find all the $N_{\max }\left(N_{\max }+2\right)\left(g_{n}^{m}, h_{n}^{m}\right)$ coefficients of the internal field that minimize the following quantity:

$$
\chi^{2}=\sum_{i=1}^{K}\left(B_{i}^{\mathrm{mes}}-B_{i}^{\mathrm{mod}}\right)^{2} \omega_{i}
$$

Given that in the POGO case we are dealing with only scalar data, the inverse problem is nonlinear and the solution to the problem is therefore not unique. It is then necessary to linearize the problem around an initial given solution. Recall that in addition, the resulting solution is contaminated by the error caused by the Backus effect (Backus, 1970, 1974). To reduce this effect, we have constrained the solution by imposing the geomagnetic equator position, estimated from different periods of modeling (Ultré-Guérard et al., 1998; Holme et al., 2005). Furthermore, considering that our aim is to describe the secular variation from these core field models, they are computed to a maximum degree and order 10 .

Minimizing the external field contributions in geomagnetic models requires data selection depending on the geomagnetic field activity, quantified by global magnetic indices such as $K_{\mathrm{p}}$ and $D_{\text {st }}$ (Mayaud, 1980; Menvielle and Berthelier, 1991). Only observations made during the time intervals with $K_{\mathrm{p}}<1$ have been kept for the present study. With such a drastic selection criteria, it is necessary to ensure that the selected data still maintain a global coverage of the Earth's surface (Fig. 4) in order to derive a good field modeling and resolve all the harmonics. It is easy to note that for OGO-2 and OGO-4 satellites (Figs. 4(a) and $4(\mathrm{~b}))$, the data coverage remains adequate even for the most restrictive planetary index $\left(K_{\mathrm{p}}=0\right)$. On the other hand (Fig. 4(c)), the OGO-6 data distribution becomes very sparse for the year 1971, even for a less restrictive option $\left(K_{\mathrm{p}}=1\right)$.

The Gauss coefficients obtained from an uneven distribution are characterized by large errors (Alexandrescu et al., 1994). The last row of Table 1 indicates the gain in the coefficient estimations when the coverage is improved. A trade- 
(a)
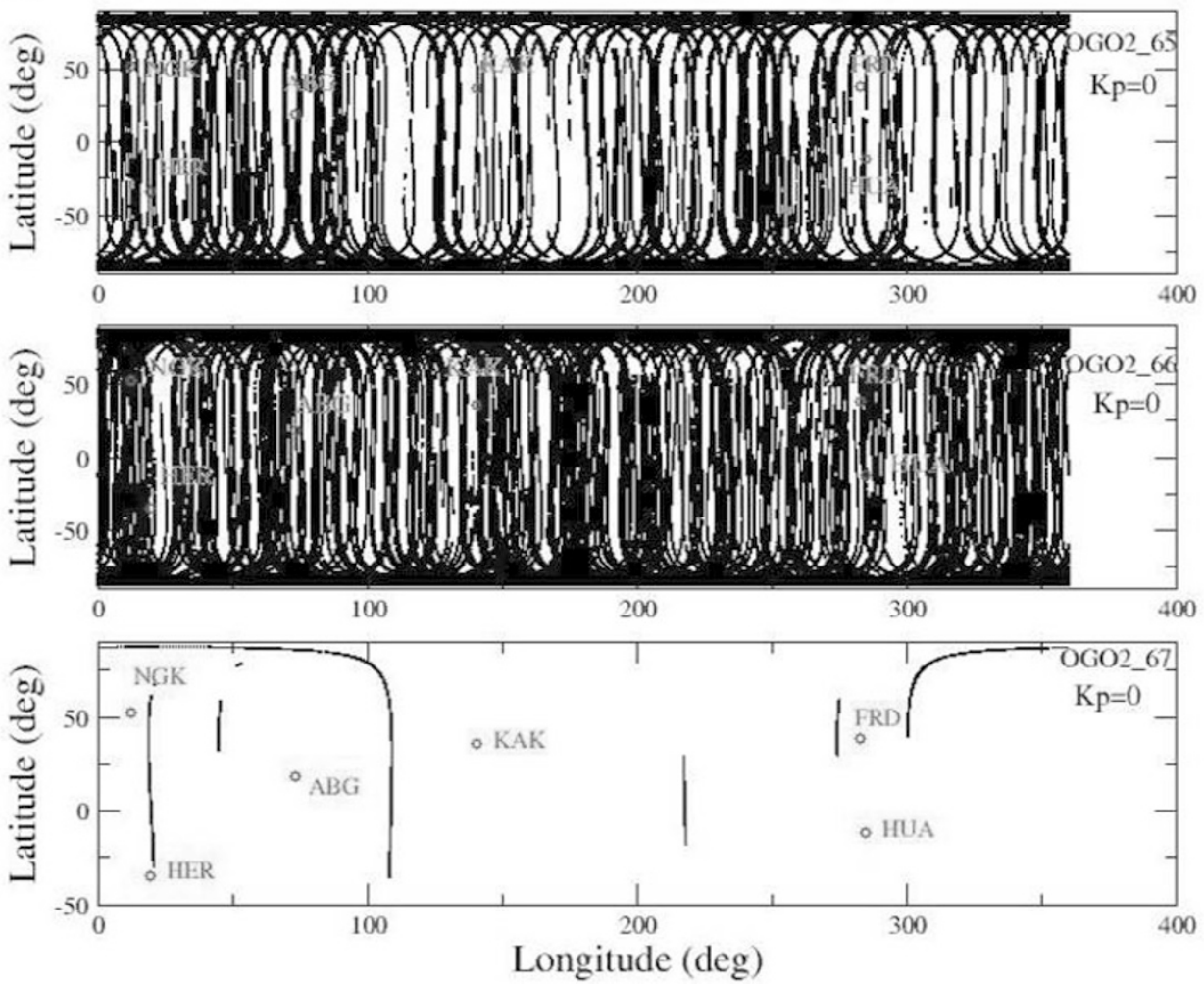

(b)
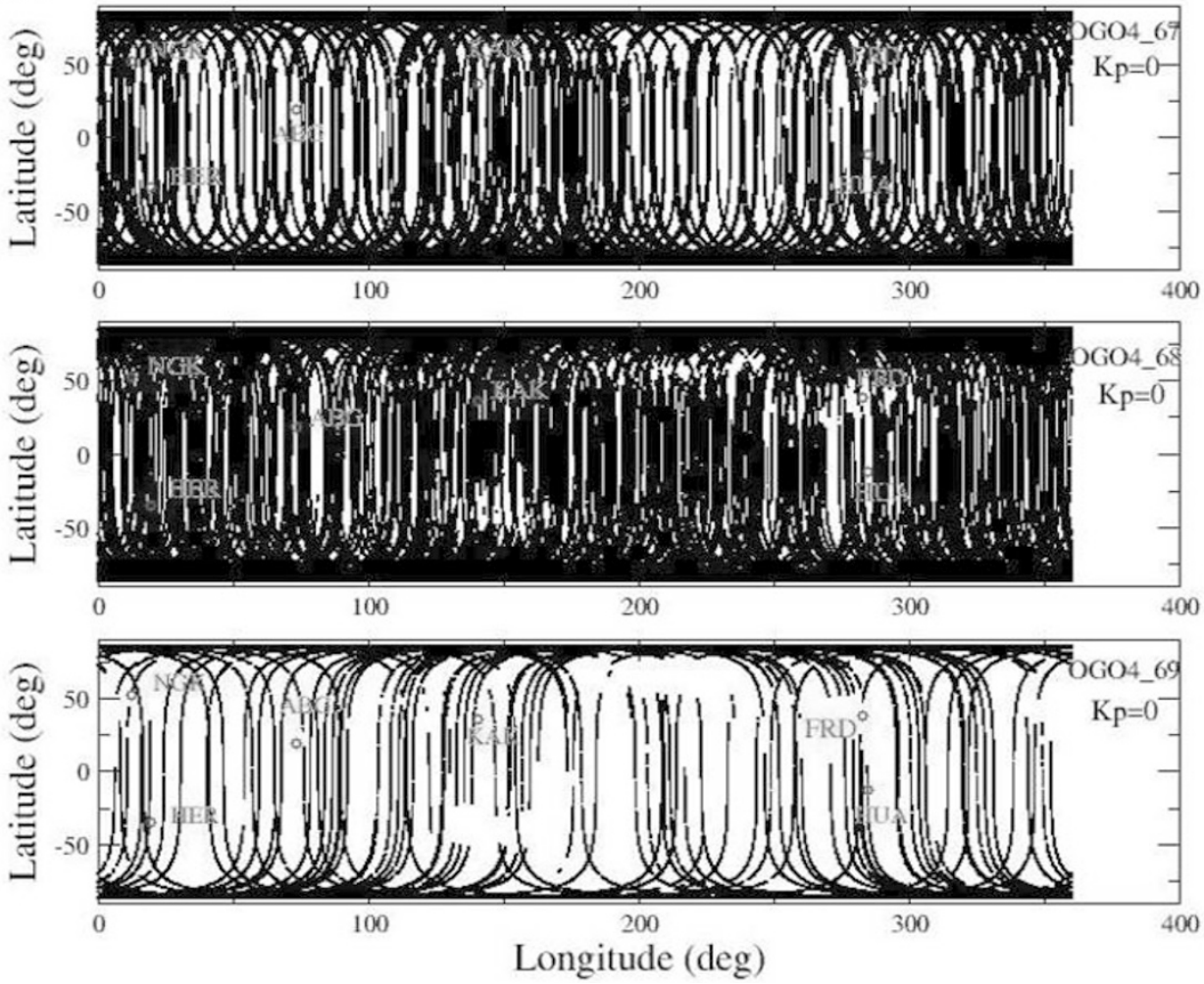

Fig. 4. Geographical distribution of the POGO measurements for the time-interval 1965-1971: (a) OGO-2, (b) OGO-4, (c) OGO-6.

off must be found between the coverage and optimal tolerated external activity. Thus, in the case of OGO-2 data, if the inversion is achieved without data selection and without knowledge of the field direction, then the model is established after only two iterations, but with a root mean square
(RMS) of some $250 \mathrm{nT}$. Still without imposing the geomagnetic equator position, but by selecting data for $K_{\mathrm{p}}=0$, the RMS value decreases to only $5 \mathrm{nT}$ at the fourth iteration. Constraining the model by the giving geomagnetic equator locations, supposedly as a priori known, the conver- 
(c)
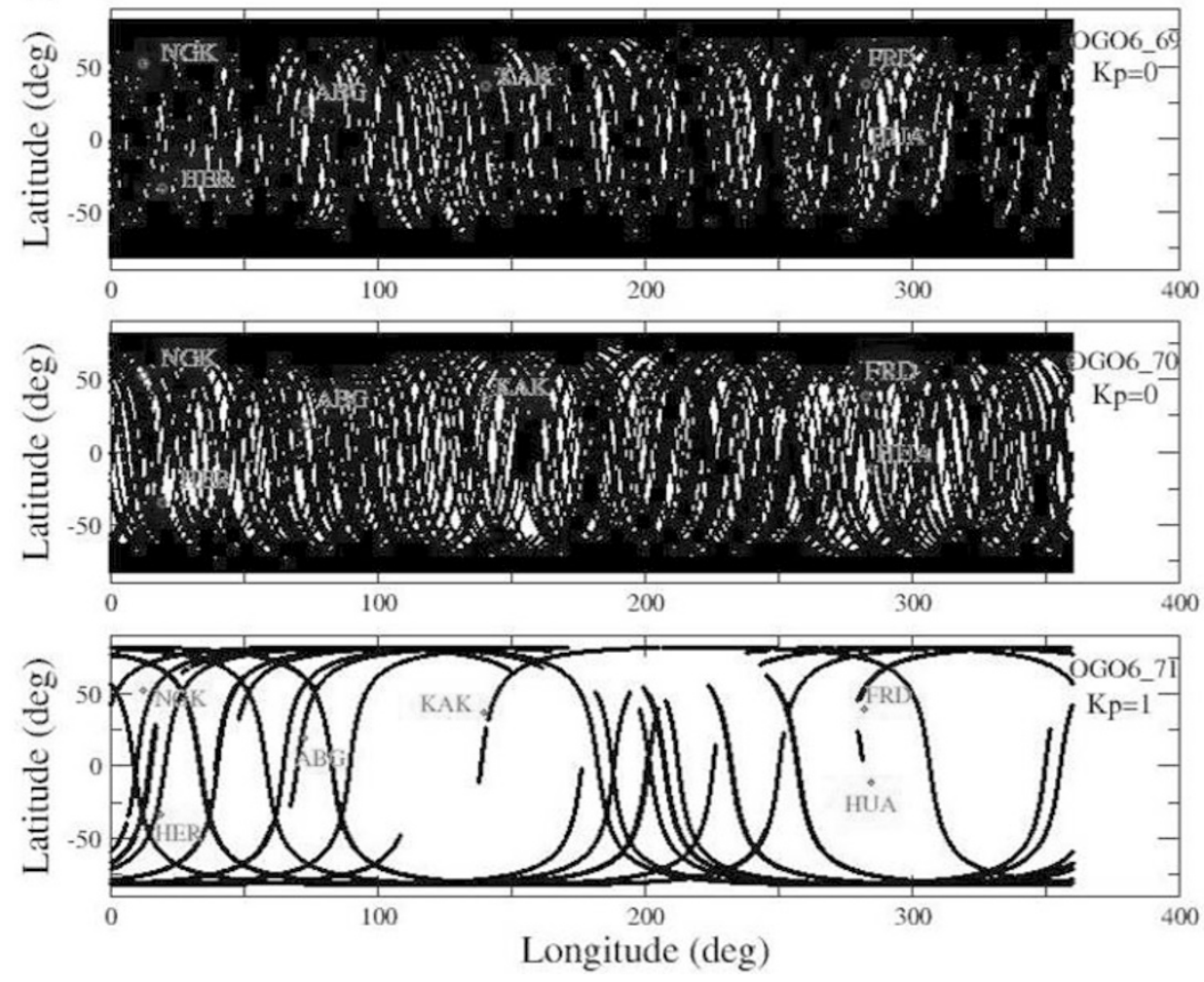

Fig. 4. (continued).

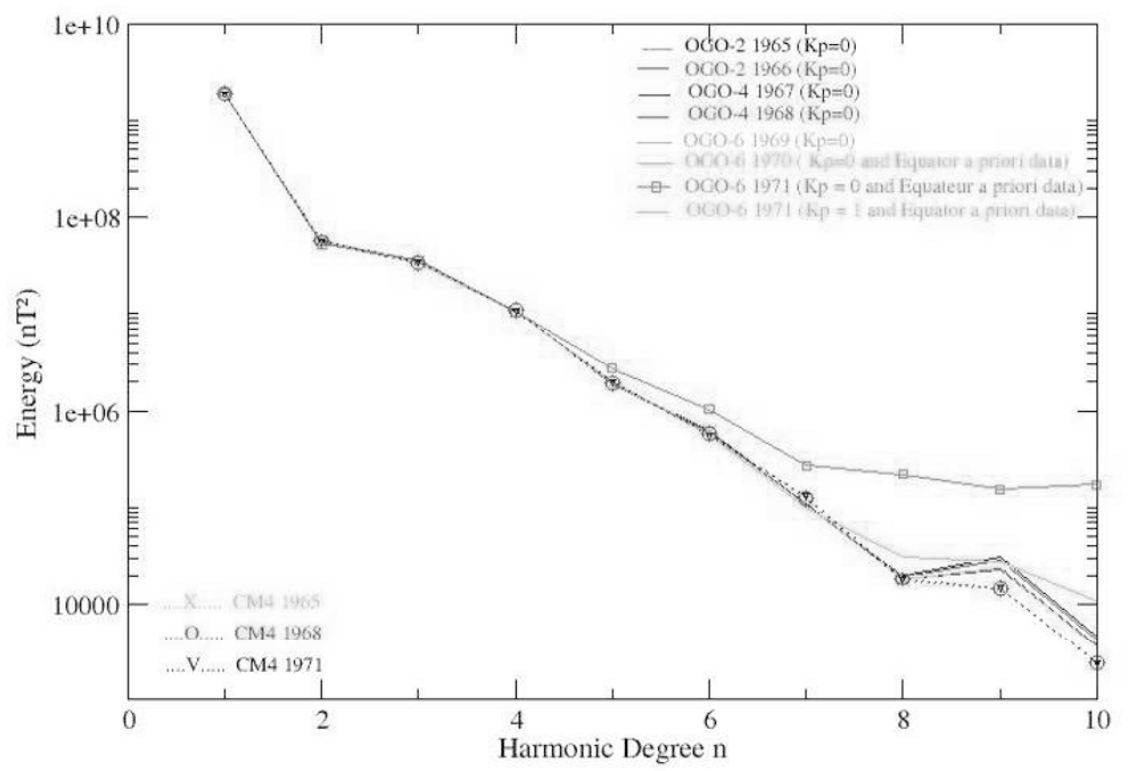

Fig. 5. Power spectra for the computed and selected POGO models. For intercomparison reasons, three power spectra for the epochs $1965,1968,1971$ based on the CM4 model are also shown.

gence towards the solution requires two iterations. Generally, the RMS is of the order of $40 \mathrm{nT}$, excepting for the very poor distribution of OGO-2 satellite measurements in 1967, when for $K_{\mathrm{p}}=0$ the global coverage is not achieved. Table 1 also includes the RMS values for all calculated models. We can note that in this table two models are avail- able for the epochs 1967 and 1969 (indeed, OGO-2 and OGO-4, and OGO-4 and OGO-6, respectively). In these two specific situations we have selected the model based on a larger available number of OGO-4 measurements (for the 1967), having also a smaller RMS. For the 1969 model the choice has been made by considering the data coverage, as 


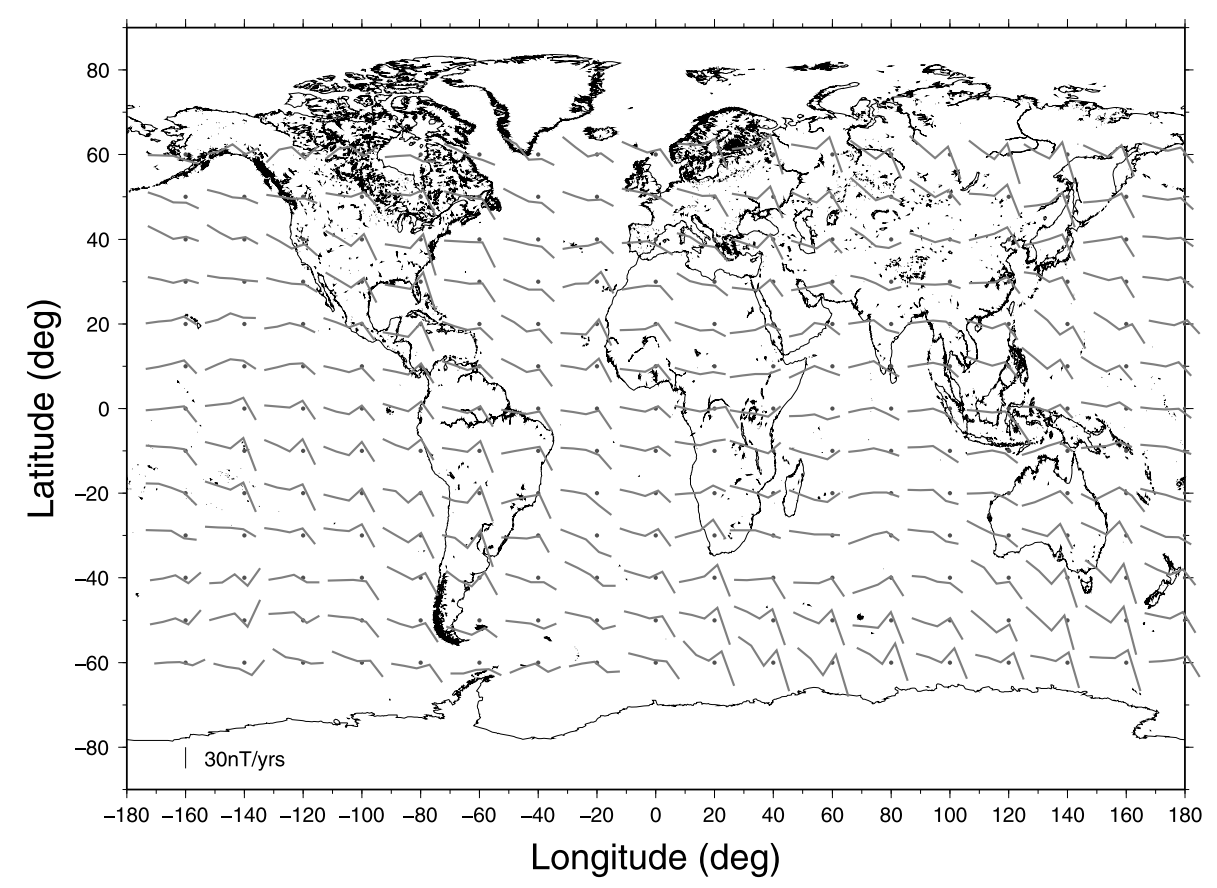

Fig. 6. Secular variation derived from POGO spherical harmonic models, estimated on a $10^{\circ} \times 20^{\circ}$ grid. The starting point for each curve is 1965 , the ending point is 1971 .

the RMS for OGO-4 and OGO-6 are slightly comparable.

For the seven selected core field models, power spectra (Lowes, 1966, 1974), have been computed and represented on Fig. 5. They are consistent with each other, exception being the model derived from OGO-6 measurements, with a poor data distribution in $1971\left(K_{\mathrm{p}}=0\right)$. For intercomparison, on the same figure, three power spectra computed from the CM4 model (Sabaka et al., 2004) are also shown, for epochs of the beginning of the interval (1965), end of the interval (1971), and middle (1968). For these epochs the core field has been computed up to degree/order 10, the same as for the POGO models. The POGO and CM4 spectra have comparable energies for the first eight degrees.

3.2.2 Application for the secular variation The POGO models, computed up to degree/order 10 have thereafter been used to synthesize the field at the chosen observatory locations. The field intensity is estimated for each year between 1965 and 1971. The values obtained are compared to those derived from the CM4 model (Sabaka et al., 2004). The secular variation of the field intensity, derived from POGO models clearly shows a change of regime in the vicinity of 1969 for the considered locations. So, we have generalized our study to the whole globe and build a map showing the annual secular variation for the total field, derived from the POGO models (Fig. 6). On this map the estimated secular variation is shown on a grid of $10^{\circ} \times 20^{\circ}$, between $-60^{\circ}$ to $60^{\circ}$ latitude, covering the time interval 1965-1971. The advantage of using only POGO measurements comes from the fact that these series of models show secular variation characteristics observed only from space. This map clearly shows changes in the secular variation trend around 1969. An interesting attempt is now to search for this jerk distribution: is it global or regional? In some region the $\mathrm{V}$-shape in secular variation is not or slightly observable, as in center Indian Ocean or Northern Pacific. These regions cover small parts of the Globe, so we cannot conclude that they are free-jerk areas.

To better underline this behaviour we also stack all curves obtained for the grid shown in Fig. 6. For comparison, for the same grid, we have estimated the secular variation from the CM4 model. In Fig. 7, the secular variation based on POGO and CM4 models is plotted, for two specific areas. The one situated in the Northern hemisphere covers 30 $60^{\circ} \mathrm{N}$ and $0-80^{\circ} \mathrm{E}$, while the one selected in the Southern hemisphere is situated over $40-60^{\circ} \mathrm{S}$ and $100-180^{\circ} \mathrm{E}$. The POGO secular variation clearly shows a change in regime around 1969, whereas this is not clear with the only six years of secular variation derived from CM4. This is the case for the full grid, and might express the fact that the CM4 model probably smoothes the rough edges of the short timescale, of one year, in the secular variations. This could be, on the one hand, linked to the used dataset by Sabaka et al. (2004), more complete than that from POGO, however also much more heterogeneous (satellites together with observatories), and on the other hand, probably because of the adjustment of the secular variation by B-cubic splines on 2.5 years nodes.

\section{Discussion and Conclusion}

The temporal behavior of Earth's magnetic field and its secular variation, have been repeatedly studied from both the ground observatory and satellite data. Our study focused on ancient satellite data, in order to extract more information on temporal evolution of the Earth's magnetic field from POGO satellite data covering a period of about seven years. We are aware of the large range of problems arising when ancient POGO data are investigated. Moreover, the data provided by the POGO missions show large variations 


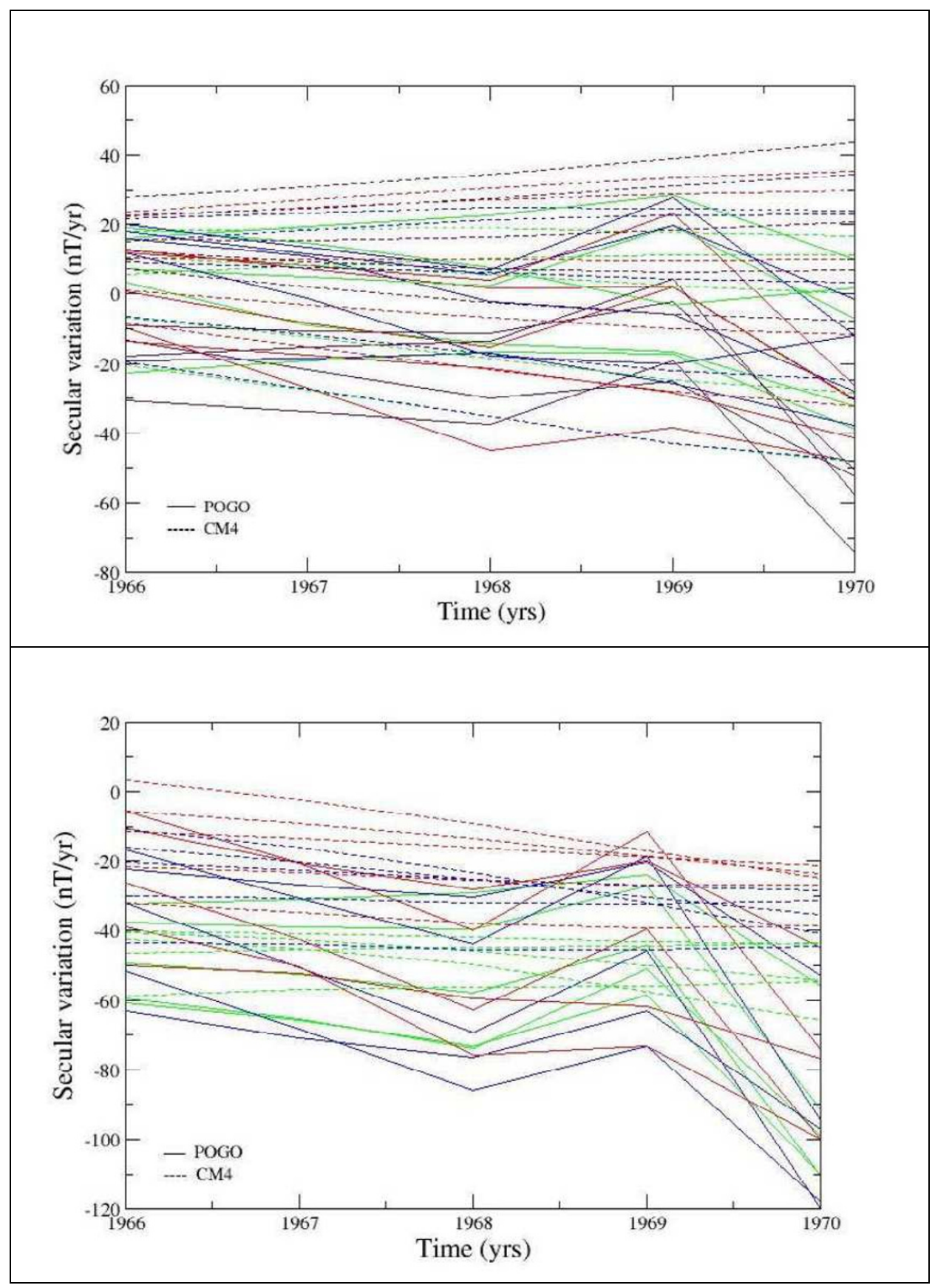

Fig. 7. The secular variation stacking curves obtained from POGO models and from CM4 model, for two specific areas of the grid given in Fig. 6. The zone selected in the Northern hemisphere is situated between $30-60^{\circ} \mathrm{N}$ and $0-80^{\circ} \mathrm{E}$ (top panel); the one selected in the Southern hemisphere is situated between $40-60^{\circ} \mathrm{S}$ and $100-180^{\circ} \mathrm{E}$ (bottom panel).

in the magnetic field intensity, due mainly to the large variations in the satellite altitude. Consequently, the variation of the field intensity remains also large, even when constraining the altitude parameter to some $100 \mathrm{~km}$ fixed-bands. We also comprehend that positioning errors represent an important error source of the magnetic intensity data of the POGO satellite series, as a position error of some $100 \mathrm{~m}$ in radial direction results in an intensity error of order of a few nT.

Nevertheless, the comparison of the satellite data averaged along each independent orbit and observatory monthly means, for each studied location, shows similarities. This supports the approach by Mandea and Olsen (2006), indicating that it is also useful to be applied to the scalar data of less accuracy than the ones provided by the Oersted or CHAMP missions. The search for trend changes in the temporal variation of the magnetic field, calculated from POGO models, is encouraging, as it shows an event around 1969, for the different selected locations. Moreover, the extension of our analysis on the global scale confirms the presence of this change in the secular variation trend. However, our attempt to find out if this geomagnetic jerk is global or regional in distribution has not been successful. We believe that more efforts have to be done and re-visit these data in order to understand the changes in the magnetic field at POGO epoch. 
Acknowledgments. We thank magnetic observatories and national agencies for efforts in producing and archiving magnetic data. Angelo De Santis and an anonymous referee are warmly acknowledged for their constructive comments.

\section{References}

Alexandrescu, M., Database of geomagnetic observatory monthly means seeks contributors, Eos AGU, 79, 345, 1998.

Alexandrescu, M., C. Ha Duyen, and J.-L. Le Mouël, Geographical distribution of magnetic observatories and field modelling, J. Geomag. Geoelectr., 46, 891-901, 1994.

Alexandrescu, M., D. Gibert, G. Hulot, J.-L. Le Mouël, and G. Saracco, Detection of geomagnetic jerks using wavelet analysis, J. Geophys. Res., 100, 12557-12572, 1995.

Alexandrescu, M., D. Gibert, G. Hulot, J.-L. Le Mouël, and G. Saracco, Worldwide analysis of geomagnetic jerk, J. Geophys. Res., 101, 2197521994, 1996.

Backus, G. E., Non-uniqueness of the external geomagnetic field determined by surface intensity measurements, J. Geophys. Res., 75, 63376341, 1970.

Backus, G. E., Determination of the external geomagnetic field determined from intensity measurements, Geophys. Res. Lett., 1, 21, 1974.

Bloxham, J., S. Zatman, and M. Dumberry, The origin of geomagnetic jerks, Nature, 420, 65-68, 2002.

Cain, J. C., R. A. Langel, and S. J. Hendricks, First magnetic field results from the OGO-2 satellite, Goddard Space Flight Center NASA Report X-612-66-305, 1966.

Cain, J. C., S. J. Hendricks, and R. A. Langel, A proposed model for the international geomagnetic reference field-1965, Goddard Space Flight Center NASA Report X-67-173, 1967.

Chambodut, A. and M. Mandea, Evidence for geomagnetic jerks in comprehensive models, Earth Planets Space, 57(2), 139-149, 2005.

Chambodut, A., C. Eymin, and M. Mandea, Geomagnetic jerks from the Earth's surface to the top of the core, Earth Planets Space, 59, 675-684, 2007.

Chapman, S. and J. Bartels, Geomagnetism, 2, 1049 pp., Oxford Univ. Press, New York, 1940.

Chulliat, A. and K. Telali, World monthly means database project, Publ. Inst. Geophys. Pol. Acad. Sci., C-99(398), 2007.

Courtillot, V., J. Ducruix, and J.-L. Le Mouël, Sur une variation récente de la variation séculaire du champ magnétique terrestre, C. R. Acad. Sci. D, 287, 1095-1098, 1978.

Gonzalez, W., J. Joselyn, Y. Kamide, H. Kroehl, G. Rostoker, B. Tsurutani, and V. Vasyliunas, What is a geomagnetic storm?, J. Geophys. Res., 99(A4), 5771-5792, 1994.

Hamoudi, M., E. Thébault, V. Lesur, and M. Mandea., GeoForschungsZentrum Anomaly Magnetic Map (GAMMA): A candidate model for the World Digital Magnetic Anomaly Map., Geochem. Geophys. Geosyst.,
8, Q06023, doi:10.1029/2007GC001638, 2007.

Holme, R., M. A. James, and H. Lühr, Magnetic field modelling from scalar-only data: Resolving the Backus effect with the equatorial electrojet, Earth Planets Space, 57, 1203-1209, 2005.

Kane, R. P., Comparison of geomagnetic changes in India and the POGO data, J. Atmos. Terr. Phys., 35, 1249-1252, 1973.

Kim, H. R. and S. D. King, A study of local time and longitudinal variability of the amplitude of the equatorial electrojet observed in POGO satellite data, Earth Planets Space, 51, 373-381, 1999.

Le Huy, M., M. Mandea, J.-L. Le Mouël, and A. Pais, Time evolution of the fluid flow at the top of the core, Earth Planets Space, 52, 163-173, 2000

Lowes, F. J., Mean-square values on sphere of spherical harmonic vector fields, J. Geophys. Res., 71, 2179, 1966.

Lowes, F. J., Spatial power spectrum of the main geomagnetic field, and extrapolation to the core, Geophys. J. R. Astron. Soc., 36, 717-730, 1974

Mandea, M. and N. Olsen, A new approach to directly determine the secular variation from magnetic satellite observations, Geophys. Res. Lett., 33, L15306, doi:10.1029/2006GL026616, 2006.

Mandea, M. and M. Purucker, Measurements of the Earth's magnetic field from space, Surv. Geophys., 26(4), 415-459, doi:10.1007/s10712-0053857-x, 2005.

Mandea, M., E. Bellanger, and J.-L. Le Mouël, A geomagnetic jerk for the end of the 20th century?, Earth Planet. Sci. Lett., 183, 369-373, 2000.

Mandea, M., M. Korte, D. Mozzoni, and P. Kotzé, The magnetic field changing over the southern African continent: a unique behaviour, South Af. J. Geol., 110(2-3), doi:10.2113/gssajg.110.2-3.193, 193-202, 2007.

Mayaud, P. N., Derivation, Meaning and Use of Geomagnetic Indices, Geophysical Monograph 22, AGU, Washington D.C., 1980.

Menvielle, M. and A. Berthelier, The K-derived planetary indices: description and availability, Rev. Geophys., 29(3), 415-432, 1991.

Olsen, N. and M. Mandea, Investigation of secular variation impulse using satellite data: the 2003 geomagnetic jerk, Earth Planet. Sci. Lett., 255, 94-105, doi:10.1016/j.espl.2006.12.008, 2007.

Sabaka, T. J., N. Olsen, and R. A. Langel, A comprehensive model of the quiet-time, near earth magnetic field: phase 3, Geophys. J. Int., 151, 32 $68,2002$.

Sabaka, T. J., N. Olsen, and M. E. Purucker, Extending comprehensive models of the earth's magnetic field with OERSTED and CHAMP data, Geophys. J. Int., 159(2), 521-547, 2004.

Ultré-Guérard, P., M. Hamoudi, and G. Hulot, Reducing the Backus effect given some knowledge of the dip-equator, Geophys. Res. Lett., 25, 3201-3204, 1998.

Y. Yahiat (e-mail: yasminayahiat@yahoo.fr), M. Hamoudi, and M. Mandea 